TRANSACTIONS OF THE

AMERICAN MATHEMATICAL SOCIETY

Volume 359, Number 11, November 2007, Pages 5125-5139

S 0002-9947(07)03402-2

Article electronically published on June 4, 2007

\title{
EXISTENCE OF OSCILLATING SOLUTIONS OF EINSTEIN SU(2) YANG-MILLS EQUATIONS
}

\author{
ALEXANDER N. LINDEN
}

\begin{abstract}
We give a rigorous proof that for small positive values of the cosmological constant the Einstein equations coupled to an SU(2) Yang-Mills connection yield oscillating spacetimes. These are static, spherically symmetric spacetimes that have the same topology as particle-like spacetimes but differ in geometry. We also give a strict upper bound on values of the cosmological constant that admit such spacetimes.
\end{abstract}

\section{INTRODUCTION}

1.1. Background. Ever since Schwarzschild found his solution of Einstein's equations in 1916 it has been known that Einstein's equations in vacuum yield no spherically symmetric static solutions that are smooth at the origin. Nor do Einstein's equations coupled to Maxwell's equations. Moreover, in both these systems the singularity at the origin is a true geometric singularity; one that cannot be removed by a change in coordinates. Thus, it came as a great surprise in the late 1980s when Bartnik and McKinnon discovered static spherically symmetric solutions of the Einstein SU(2) Yang-Mills equations that are not only smooth at the origin, but are globally smooth 1 .

Smoller, Wasserman, Yau, and McLeod gave the first rigorous existence proof of these solutions [8]. They analyzed the equations in Schwarzschild coordinates $(t, r, \phi, \theta)$; coordinates in which a static spherically symmetric metric assumes the following form:

$$
\mathrm{d} s^{2}=-c^{2} C^{2}(r) A(r) \mathrm{d} t^{2}+\frac{1}{A(r)} \mathrm{d} r^{2}+r^{2}\left(\mathrm{~d} \phi^{2}+\sin ^{2} \phi \mathrm{d} \theta^{2}\right),
$$

where $c$ is constant (the speed of light). They proved that solutions exist in which the metric (10) is smooth for all $r \in[0, \infty)$.

In the same paper they proved the existence of a 1-parameter family of solutions that are smooth at $r=0$. Smoller and Wasserman subsequently proved that this family contains a countable subfamily of global smooth solutions 9]. Brietenlohner, Forgács, and Maison later classified all solutions in the 1-parameter family [3]. In particular, they proved that each solution must be one of three types.

Generic: The underlying spacetime has the topology of $\mathbb{R} \times S^{3}$. In appropriate coordinates one pole of each constant-time spacelike 3 -sphere is the center of spherical symmetry and the opposite pole has a Reissner-Nordström-like singularity. There is a horizon at the equator where the radius is maximum. Such solutions

Received by the editors July 27, 2001 and, in revised form, October 8, 2002.

2000 Mathematics Subject Classification. Primary 83C20.

(C)2007 American Mathematical Society Reverts to public domain 28 years from publication 
are generic in the sense that their qualitative behavior does not change with small static spherically symmetric perturbations.

Particle-like: These are the aforementioned global smooth solutions. A particle-like solution is defined to be a solution that is global and smooth in Schwarzschild coordinates; i.e., $A(r)$ and $C(r)$ of metric (1) are smooth and nonzero for all $r \geq 0$. Particle-like solutions also have the property that as $r \nearrow \infty$, the metric approaches that of Minkowski space and the Yang-Mills field vanishes 8 .

Oscillating: As with particle-like solutions, each constant-time hypersurface has the topology of $\mathbb{R}^{3}$. However, the coordinate $r$ of the Schwarzschild coordinate system cannot be extended to infinity. Rather, any radial geodesic parameterized by $r$ has infinite length over a finite range of $r$. More importantly, whereas particlelike spacetimes are asymptotically flat, oscillating spacetimes are not. Oscillating solutions also exhibit a different behavior in the Yang-Mills potential. They are called oscillating because of the asymptotic behavior of the Yang-Mills potential.

The analyses [8], [9], and [3] all presupposed the absence of a cosmological constant. The addition of a cosmological term to Einstein's equations in the static spherically symmetric Einstein SU(2) Yang-Mills system does not affect the behavior of solutions for small $r$ 6]. In particular, there still exists a 1-parameter family of solutions that are smooth at the origin. However, a positive cosmological term precludes the existence of particle-like solutions. Specifically, for any solution expressed in Schwarzschild coordinates that is smooth at the origin, there exists a finite $r_{c}$ (which depends on the solution) such that $\lim _{r} / r_{c} A(r)=0$ 4. This causes the metric to become singular, at least in Schwarzschild coordinates.

The question arises as to the nature of this singularity. Is it a true geometric singularity or only due to the choice of coordinates? Reference [7] examines all of the types of singularities at $r_{c}$ that are possible. In the case of positive cosmological constant they are as follows:

Generic singularity. A solution has a generic singularity if there exists a change in coordinates from Schwarzschild coordinates $(t, r, \phi, \theta)$ to new coordinates $(t, \tau, \phi, \theta)$, such that in the new coordinates

(1) the metric is smooth at $\left(t, \tau_{c}=\tau\left(r_{c}\right), \phi, \theta\right)$, and

(2) for each $(t, \phi, \theta)$ there exists a neighborhood $U$ of the set $\left\{\left(t, \tau_{c}, \phi, \theta\right)\right\}$ such that $r_{c}$ is a unique maximum of $r$ on $U$.

This is similar to the singularity that occurs in the metric on $\mathbb{R}$ described by $\mathrm{d} s^{2}=$ $\left(1-r^{2}\right)^{-1} \mathrm{~d} r^{2}$ at $r=1$. Indeed, the coordinate change $\tau=\sin ^{-1} r$ removes the singularity.

Oscillating singularity. A solution has an oscillating singularity whenever the singularity at $r_{c}$ is not a singularity of the metric but apparent only because a finite range of the coordinate $r$ covers the entire manifold. The metric $\mathrm{d} s^{2}=\sec ^{4} r \mathrm{~d} r^{2}$ on $\mathbb{R}$ has similar "singularities" at $r= \pm \pi / 2$. Indeed, the coordinate change $\tau=\tan r$ transforms the metric to $\mathrm{d} s^{2}=\mathrm{d} \tau^{2}$.

Noncompact singularity. A solution has a noncompact singularity whenever the singularity can be removed by a Kruskal-like change in coordinates; i.e., the singularity is of the same nature as the coordinate singularity of the Schwarzschild solution of Einstein's equations in vacuum.

This classification of singularities does not require solutions to be smooth at the origin. Thus, the inevitable question is which of these singularities can be realized by solutions that are smooth at the origin. Reference [4] contains a proof that 
noncompact singularities are realized. The analysis of Brietenlohner, Forgács, and Maison together with simple perturbation techniques establishes the existence of solutions that are smooth at the origin and have a generic singularity at $r_{c}$. In this paper we prove that, provided the cosmological constant is small, there exist solutions that are smooth at the origin and have an oscillating singularity at $r_{c}$. We also provide a sharp bound on the values of $\Lambda$ that admit such solutions.

1.2. Equations, scaling, and units. We consider initially classical physical coordinates of time measured in units of $T$ and length measured in units of $L$. Gravitational mass is measured in units of $M$. Einstein's equations with positive cosmological constant $\Lambda$ written in coordinates $\left(x_{0}, x_{1}, x_{2}, x_{3}\right)$ are as follows:

$$
R_{i j}-\frac{1}{2} R g_{i j}+\Lambda g_{i j}=\frac{8 \pi G}{c^{4}} T_{i j} .
$$

System (2) is to be solved for the metric coefficients $g_{i j}=\mathrm{d} s^{2}\left(\partial / \partial x_{i}, \partial / \partial x_{j}\right) . R_{i j}$ and $R$ are respectively the Ricci curvature and scalar curvature tensors that are induced by the Riemannian connection. $G$ is Newton's gravitational constant and has units of $L^{3} /\left(M T^{2}\right), c$ is the speed of light in vacuum and has units of $L / T$, and $T_{i j}$ is the covariant stress energy tensor and has units of $M L / T^{2}$. Thus, provided the $g_{i j}$ have units of $L^{2}$ and $\Lambda$ units of $1 / L^{2}$, equations (2) are invariant under scalings of $L, T$, and $M$.

To account for the effects of scaling mathematically, corresponding to coordinates $(t, r, \phi, \theta)$, we consider scaled coordinates $\left(\sigma_{t} t, \sigma_{r} r, \phi, \theta\right)$, where $\sigma_{t}$ and $\sigma_{r}$ are arbitrary positive constants. Given another arbitrary positive constant $\sigma_{m}$, we define any function $X(t, r, \phi, \theta, m)$ to have dimension $T^{\alpha} L^{\beta} M^{\gamma}$ whenever

$$
X\left(\sigma_{t} t, \sigma_{r} r, \phi, \theta, \sigma_{m} M\right)=\sigma_{t}^{\alpha} \sigma_{r}^{\beta} \sigma_{m}^{\gamma} X(t, r, \phi, \theta, m) .
$$

$\sigma_{t}, \sigma_{r}$, and $\sigma_{m}$ should be thought of as time, length, and mass scaling constants respectively.

Next, we write a spherically symmetric metric in scaled coordinates as

$$
\begin{aligned}
\mathrm{d} s_{\text {scaled }}^{2}= & -c^{2} C^{2}\left(\sigma_{r} r\right) A\left(\sigma_{r} r\right) \mathrm{d}\left(\sigma_{t} t\right)^{2}+\frac{1}{A\left(\sigma_{r} r\right)} \mathrm{d}\left(\sigma_{r} r\right)^{2} \\
& +\left(\sigma_{r} r\right)^{2}\left(\mathrm{~d} \phi^{2}+\sin ^{2} \phi \mathrm{d} \theta^{2}\right) .
\end{aligned}
$$

Comparing the metric (3) to the metric (11) (which we view as an unscaled metric) makes evident that the coordinate $t$ has units of $T$ and the coordinate $r$ has units of $L$ whereas $C$ and $A$ are unitless. We always scale so that $\sigma_{t}=\sigma_{r}$ and denote this metric scaling factor by $\sigma_{g}$. Now $c$, being physically measurable, has units $L / T$. By assumption it is independent of $\sigma_{g}$. G, also being physically measurable, has units $L^{3} /\left(M T^{2}\right)$.

In $s u(2)$, the lie algebra of $\mathrm{SU}(2)$, without loss of generality, we take as a basis

$$
\tau_{1}=\frac{i}{2}\left(\begin{array}{cc}
0 & -1 \\
1 & 0
\end{array}\right), \quad \tau_{2}=\frac{i}{2}\left(\begin{array}{cc}
0 & i \\
i & 0
\end{array}\right), \quad \tau_{3}=\frac{i}{2}\left(\begin{array}{cc}
-1 & 0 \\
0 & 1
\end{array}\right) .
$$

$\tau_{1}, \tau_{2}, \tau_{3}$ satisfy

$$
\left[\tau_{1} \tau_{2}\right]=\tau_{3}, \quad\left[\tau_{2} \tau_{3}\right]=\tau_{1}, \quad\left[\tau_{3} \tau_{1}\right]=\tau_{2},
$$

where $[x y]=x y-y x$ by ordinary matrix multiplication. We also take a Killing inner product

$$
\langle x y\rangle_{s}=-\frac{2 \sigma_{s}^{2} e}{G} \operatorname{trace}(\operatorname{ad} x \operatorname{ad} y),
$$


where $\operatorname{ad} x \in \operatorname{gl}(2, \mathbb{C})$ is defined by ad $x(y)=\left[\begin{array}{ll}x & y\end{array}\right] . \sigma_{s}$ is an arbitrary positive scaling constant, $e$ is an arbitrary positive constant (the coupling constant), and $G$ is, as before, Newton's gravitational constant.

We consider a general spherically symmetric Yang-Mills magnetic only potential on the yet to be determined manifold with scaled metric (3). In coordinates $\left(\sigma_{g} t, \sigma_{g} r, \phi, \theta\right)$ this connection is as follows:

$$
\omega_{Y M}=\mathrm{w}\left(\sigma_{g} r\right) \tau_{2} \mathrm{~d} \phi+\left(\cos \phi \tau_{3}-\mathrm{w}\left(\sigma_{g} r\right) \sin \phi \tau_{1}\right) \mathrm{d} \theta .
$$

It is clear that $\mathrm{w}$ has no dimension of time or length. We assume it to have no dependence on mass so that $\mathrm{w}$ is dimensionless. We also assume elements of $s u(2)$ to have no dependence on mass. Thus, the $\tau_{i}$ are also dimensionless. We assume $\sigma_{s}$ to be a length scale. Then, since an inner product scales by $L^{2}$, equation (4) shows that $e$ must have dimension $L^{2}$ times the dimension of $G$; i.e., $e$ has dimension $L^{5} / M T^{2}$.

We define

$$
F=\mathrm{D} \omega_{Y M}
$$

where $\mathrm{D}$ is the covariant derivative with respect to the Yang-Mills connection. The Yang-Mills equations are

$$
\mathrm{D} * F=0
$$

where $*$ is the Hodge star operator induced by the metric on the spacetime manifold. This metric and the metric (4) on $s u(2)$ yield a product metric $\langle,\rangle_{x}=\mathrm{d} s^{2}$. $\langle,\rangle_{s}$ on the product manifold constructed from the space of two forms on the spacetime manifold and $s u(2)$. The stress energy tensor, expressed in coordinates that diagonalize the metric (3) at a point, is then determined by

$$
T_{i j}=\frac{\partial}{\partial g^{i j}}\left(\langle F, F\rangle_{x}\right)-\frac{g_{i j}}{2}\langle F, F\rangle_{x} .
$$

The coupled Einstein SU(2) Yang-Mills equations become

$$
\left(\sigma_{g} r\right) A^{\prime}+2 \frac{e}{G} \sigma_{s}^{2} A w^{\prime 2}=1-A-\frac{e}{G} \sigma_{s}^{2} \frac{\left(1-\mathrm{w}^{2}\right)^{2}}{\left(\sigma_{g} r\right)^{2}}-\Lambda g\left(\sigma_{g} r\right)^{2},
$$

$$
\begin{gathered}
\left(\sigma_{g} r\right)^{2} A \mathrm{w}^{\prime \prime}+\left(\sigma_{g} r\right) \mathrm{w}^{\prime}\left(1-A-\frac{e}{G} \sigma_{s}^{2} \frac{\left(1-\mathrm{w}^{2}\right)^{2}}{\left(\sigma_{g} r\right)^{2}}-\Lambda_{g}\left(\sigma_{g} r\right)^{2}\right)+\mathrm{w}\left(1-\mathrm{w}^{2}\right)=0, \\
\frac{C^{\prime}}{C}=\frac{e}{G} \frac{2 \sigma_{s}^{2} w^{\prime 2}}{\sigma_{g} r}
\end{gathered}
$$

where ' denotes $\frac{\mathrm{d}}{\mathrm{d}\left(\sigma_{g} r\right)}=\frac{1}{\sigma_{g}} \mathrm{~d} r$ and $\Lambda_{g}$ is arbitrary. If $\sigma_{g}=\sigma_{s}$, then these equations simplify to

$$
\begin{gathered}
r A^{\prime}+\frac{e}{G} 2 A \mathrm{w}^{\prime 2}=1-A-\frac{e}{G} \frac{\left(1-\mathrm{w}^{2}\right)^{2}}{r^{2}}-\Lambda r^{2}, \\
r^{2} A \mathrm{w}^{\prime \prime}+r \mathrm{w}^{\prime}\left(1-A-\frac{e}{G} \frac{\left(1-\mathrm{w}^{2}\right)^{2}}{r^{2}}-\Lambda r^{2}\right)+\mathrm{w}\left(1-\mathrm{w}^{2}\right)=0, \\
\frac{C^{\prime}}{C}=\frac{e}{G} \frac{2 \mathrm{w}^{\prime 2}}{r}
\end{gathered}
$$


where $\Lambda=\Lambda_{g} / \sigma_{g}^{2}$ and ' denotes $\frac{\mathrm{d}}{\mathrm{d} r}$; i.e., the system of equations (8), (9), (10) is scale invariant provided the Yang-Mills metric and spacetime metric scale by the same factor.

To geometrize the units we set $c=G=1$. This forces units of $T$ and $M$ which are determined by units of $L$. We then set $e=1$ which forces units of $L$ which depend only on the relative strength of the gravitational and Yang-Mills forces; i.e., one unit of length equals $\sqrt{e / G}$.

Throughout the rest of this paper, we assume that $r$ and $\Lambda$ are measured in these units. Consequently, we analyze the following equations that are satisfied by the dimensionless $A(r), C(r)$ and $\mathrm{w}(r)$ defined by metric (1) and Yang-Mills connection (5):

$$
\begin{gathered}
r A^{\prime}+2 A \mathrm{w}^{\prime 2}=\Phi, \\
r^{2} A \mathrm{w}^{\prime \prime}+r \mathrm{w}^{\prime} \Phi+\mathrm{w}\left(1-\mathrm{w}^{2}\right)=0, \\
\frac{C^{\prime}}{C}=\frac{2 \mathrm{w}^{\prime 2}}{r},
\end{gathered}
$$

where

$$
\Phi=1-A=\frac{\left(1-\mathrm{w}^{2}\right)^{2}}{r^{2}}-\Lambda r^{2} .
$$

Because equation (14c) separates from equations (14a) and (14b), we neglect it.

\section{OutLine}

We consider solutions of equations (14a) -114b) that are members of the 1parameter family of solutions that are smooth at the origin. For any such solution, we have

$$
\left(A(0), \mathrm{w}(0), \mathrm{w}^{\prime}(0), \mathrm{w}^{\prime \prime}(0)\right)=(1,1,0,-\lambda) ;
$$

$\lambda$ being the parameter of the family [8], 6]. Our goal is to establish the existence of solutions in this family that have an oscillating singularity. To be rigorous, we define an oscillating solution independently of $\Lambda$ precisely as follows:

Definition. A solution $(A(r), \mathrm{w}(r))$ of the Einstein $\mathrm{SU}(2)$ Yang-Mills equations (14a) - (14b) is called an oscillating solution if the following hold:

(1) $(A(r), \mathrm{w}(r))$ satisfies the conditions of equation (16),

(2) w has an infinite number of zeros in a finite interval $\left(0, r_{c}\right)$,

(3) with the solution $(A, \mathrm{w})$, equations (14a)-(14b) are nonsingular for all $r \in$ $\left(0, r_{c}\right)$.

We shall introduce a new parameter $\tau$ to replace the coordinate $r$ and, with $\tau$, some new variables. Oscillating solutions will then be shown to have a nice characterization in terms of the behavior of these new variables. This characterization is the content of Theorem 1. Theorem 1 and perturbation arguments will then be used to prove the existence of oscillating solutions when $\Lambda$ is small. This is the content of Theorem 3 . 


\section{EXISTENCE PROOFS}

Given any $(\Lambda, \lambda) \in \mathbb{R}^{2}$, we write $x(\Lambda, \lambda)$ or $x(\Lambda, \lambda, r)$ for any variable $x$ derived from the solution of equations (14a) - 114b) with cosmological constant $\Lambda$ that satisfies the conditions of equation (16) with $\lambda=-\mathrm{w}^{\prime \prime}(0)$. For any such solution, $(A(\Lambda, \lambda), \mathrm{w}(\Lambda, \lambda))$, the crash point $r_{c}(\Lambda, \lambda)$ is defined as follows:

$$
\begin{aligned}
& r_{c}(\Lambda, \lambda)=\infty \quad \text { whenever }(A(\Lambda, \lambda), \mathrm{w}(\Lambda, \lambda)) \text { is particle-like, } \\
& r_{c}(\Lambda, \lambda)=\min \{\tilde{r}>0: A(\Lambda, \lambda, \tilde{r})=0\} \quad \text { otherwise. }
\end{aligned}
$$

Results in [7, 4, and [8] imply that $r_{c}$ is well defined for all $\Lambda \geq 0$ and all $\lambda$. In particular, $r_{c}(\Lambda, \lambda)=\infty$ only for particle-like solutions. Moreover, for each $(\Lambda, \lambda)$, equations (14a) - 14b are nonsingular for all $r \in\left(0, r_{c}(\Lambda, \lambda)\right)$. To simplify notation, given $(\Lambda, \lambda)$, whenever a limit is established for a variable $x(\Lambda, \lambda, r)$ as $r \nearrow r_{c}$, we denote this limit by $x_{c}(\Lambda, \lambda)$ or, when the choice of $\Lambda$ is clear, by $x(\lambda)$.

As in [3, we introduce the new variables

$$
\begin{gathered}
N=\sqrt{A}, \\
U=N \mathrm{w}^{\prime}, \\
\kappa=\frac{1}{2 N}\left(\Phi+2 U^{2}+2 N^{2}\right),
\end{gathered}
$$

and define a new parameter $\tau$ by $\mathrm{d} r / \mathrm{d} \tau=r N$. Equations (14a)-114c) transform into

$$
\begin{gathered}
\dot{r}=r N, \\
\dot{\mathrm{w}}=r U, \\
\dot{N}=(\kappa-N) N-2 U^{2}, \\
\dot{U}=-\frac{\mathrm{w}\left(1-\mathrm{w}^{2}\right)}{r}-(\kappa-N) U, \\
\dot{C} N=(\kappa-N) C N,
\end{gathered}
$$

where the $\operatorname{dot}()$ here and elsewhere denotes $\mathrm{d} / \mathrm{d} \tau$. We also have the auxiliary equation

$$
\dot{\kappa}=1+2 U^{2}-\kappa^{2}-2 \Lambda r^{2} .
$$

The metric transforms into

$$
\mathrm{d} s^{2}=-c^{2} C^{2}(\tau) N^{2}(\tau) \mathrm{d} t^{2}+r^{2}(\tau)\left(\mathrm{d} \tau^{2}+\mathrm{d} \phi^{2}+\sin ^{2} \phi \mathrm{d} \theta^{2}\right) .
$$

Solutions of equations (14a) -(14b) that satisfy initial conditions (16) in the region $r \in\left(0, r_{c}\right)$ are equivalent to solutions of equations (21a) $-(21 \mathrm{f})$ that satisfy

$$
\begin{aligned}
r(-\infty) & =0, \\
N(-\infty) & =1, \\
\mathrm{w}(-\infty) & =1, \\
U(-\infty) & =0, \\
\kappa(-\infty) & =1, \\
\dot{U}(-\infty) & =-\lambda
\end{aligned}
$$

in a corresponding region $\tau \in\left(-\infty, \tau_{c}\right)$. As expected, equation (21e) separates from the others. Therefore, it can be ignored. Throughout this analysis we make no 
distinction between solutions $(A, \mathrm{w})$ of equations (14a) -14b) with initial conditions (16) and solutions $(r, N, \mathrm{w}, U)$ of equations (21a)-(21f) with initial conditions (23). For any such solution, we define

$$
\tau_{c}=\min \left\{\tau \in \mathbb{R} \mid r(\tau)=r_{c}\right\}
$$

whenever such $\tau$ exist. Otherwise, we define $\tau_{c}=\infty$.

Whereas $r_{c}=\infty$ is a sufficient condition for a solution to be particle-like, $\tau_{c}=\infty$ is not. Specifically, we have the following:

Theorem 1. Suppose $(r, N, \mathrm{w}, U)$ is a solution of equations (21a)-(21f) with $\Lambda \geq 0$ that satisfies the conditions of equations (23). Then the following are equivalent:

$\mathrm{I}:(r, N, \mathrm{w}, U)$ is oscillating.

II: (a) $r_{c}$ is finite,

(b) $\tau_{c}=\infty$.

III: (a) $\Phi_{c}=0$,

(b) $\mathrm{w}_{c}=0$

(c) $\kappa_{c}=+\sqrt{1-2 \Lambda r_{c}^{2}}$.

Proof. (II $\Rightarrow$ III). Since $\lim _{r / r_{c}} A(r)$ and $\lim _{r} / r_{c} \mathrm{w}(r)$ are both well defined [7, $\Phi_{c}=\lim _{r} / r_{c} \Phi(r)$ is well defined also. If $\Phi_{c}<0$, then equation (14a) gives $\varlimsup_{r}>r_{c} A^{\prime}(r)<0$. Consequently, there exist $\epsilon>0$ and $\delta>0$ such that $\sqrt{A}(r)>\epsilon \sqrt{r-r_{c}}$ whenever $r \in\left(r_{c}-\delta, r_{c}\right)$. Substituting this into equation (21a) and integrating gives $\tau_{c}<\infty$, contrary to hypothesis. Therefore, $\Phi_{c} \geq 0$. It follows easily from equation (14b) that, if $\Phi_{c}>0$, then $\overline{\lim }_{r} / r_{c} \mathrm{w}^{2}(r)<\infty$ which in turn implies that $\left(A \mathrm{w}^{2}\right)_{c}=0$. Since $A \searrow 0$ as $r \nearrow r_{c}$, equation (14a) then gives $\Phi_{c} \leq 0$. This establishes III(a).

To establish III(c), we notice that for any solution $(r, N, \mathrm{w}, U)$ of equations (21a)(21f), $\kappa+N$ satisfies

$$
\frac{\mathrm{d}}{\mathrm{d} \tau}(\kappa+N)=-\frac{3}{4}(\kappa-N)^{2}-\frac{1}{4}(\kappa+N)^{2}+1-2 \Lambda r^{2} \leq 1-2 \Lambda r^{2} .
$$

If, for any $\epsilon>0,2 \Lambda r_{c}^{2}=1+2 \epsilon^{2}$, then there exist $\tilde{\tau}$ such that for all $\tau>\tilde{\tau}$, $\dot{\kappa}+\dot{N}<-\epsilon^{2}$. Since $\tau_{c}=\infty, \lim _{\tau} / \infty(\kappa+N)(\tau)=-\infty$. Also, since $N>0$ for all $\tau \in \mathbb{R}$, it follows that

$$
\varlimsup_{\tau / \infty} \kappa(\tau) \leq \lim _{\tau / \infty}(\kappa+N)(\tau)-\varliminf_{\tau / \infty} N(\tau)=-\infty ;
$$

i.e., $\lim _{\tau / \infty} \kappa(\tau)=-\infty$. However, $\underline{\lim }_{\tau / \tau_{c}} \kappa(\tau)=-\infty$ implies $\tau_{c}<\infty$ [7]. Thus, $2 \Lambda r_{c}^{2} \leq 1$.

It follows immediately from equation (21f) that $\underline{\lim }_{\tau / \tau_{c}} \kappa(\tau) \geq 0$ since $\dot{\kappa}(\tilde{\tau})>0$ for any $\tilde{\tau}$ that satisfies $\kappa(\tilde{\tau})=0 . \Phi_{c}=0$ and the definition of $\kappa$ (equation (20)) give $U_{c}=0$. To complete the proof that $\kappa$ has a limit as $\tau \nearrow \infty$, we define $\kappa_{c}=\sqrt{1-2 \Lambda r_{c}^{2}}$. For any $\bar{\kappa}>\kappa_{c}$ there exist $\epsilon>0$ such that $\bar{\kappa}^{2}>\kappa_{c}^{2}+2 \epsilon$. Consequently, whenever $\kappa(\tau) \geq \bar{\kappa}$ and $\tau$ is sufficiently large, $\dot{\kappa}(\tau)<-\epsilon$. Therefore, $\varlimsup_{\tau / \infty} \kappa(\tau) \leq \kappa_{c}$. Similarly, $\underline{\lim }_{\tau / \infty} \kappa(\tau) \geq \kappa_{c}$. III(c) follows.

To establish III(b) we prove that whenever $\Phi_{c}=0$ and $\left(1-\mathrm{w}_{c}^{2}\right) \mathrm{w}_{c} \neq 0$, then $\tau_{c}<$ $\infty$. Indeed, equation (14b) implies that $\mathrm{w}^{\prime}$ has only one sign near $r_{c}$. Consequently, so does $U$. Since $U_{c}=0$, equation (21d) yields

$$
\mathrm{w}_{c} \mathrm{w}_{c}^{\prime}\left(1-\mathrm{w}_{c}^{2}\right)>0 \text {. }
$$


Without loss of generality we assume $\mathrm{w}^{\prime}$ to be positive near $r_{c}$. Now, for any solution of equations (14a)-(14b), $A \mathrm{w}^{\prime}$ satisfies

$$
r^{2}\left(A \mathrm{w}^{\prime}\right)^{\prime}+2 r \mathrm{w}^{\prime 2}\left(A \mathrm{w}^{\prime}\right)+\mathrm{w}\left(1-\mathrm{w}^{2}\right)=0 .
$$

Equation (26) gives, for $r$ near $r_{c}$,

$$
A=\frac{A \mathrm{w}^{\prime}}{\mathrm{w}^{\prime}}>\frac{\mathrm{w}_{c}\left(1-\mathrm{w}_{c}^{2}\right)\left(r_{c}-r\right)}{2 r_{c}^{2} \mathrm{w}^{\prime}} .
$$

Since $A_{c}^{\prime}=0$, it follows that $\lim _{r} \succ r_{c} \mathrm{w}^{\prime}(r)=\infty$.

Differentiating equation (14a) gives

$$
A^{\prime \prime}=\mathrm{w}^{\prime}\left[\frac{-2 A^{\prime}}{r \mathrm{w}^{\prime}}+\frac{2 \mathrm{w}^{\prime} z}{r}+\frac{2\left(1-\mathrm{w}^{2}\right)^{2}}{r^{4} \mathrm{w}^{\prime}}-\frac{2 \Lambda}{\mathrm{w}^{\prime}}+\frac{8 \mathrm{w}\left(1-\mathrm{w}^{2}\right)}{r^{3}}\right],
$$

where $z=\left(\Phi+2 A \mathrm{w}^{\prime 2}\right) / r$. Multiplying and dividing equation (20) by $\mathrm{w}^{\prime}$ gives

$$
\lim _{r \nearrow r_{c}} \mathrm{w}^{\prime} z(r)=0
$$

which, when substituted into equation (28), gives immediately that as $r \nearrow r_{c}$,

$$
A^{\prime \prime}(r) \sim \frac{8 \mathrm{ww}^{\prime}\left(1-\mathrm{w}^{2}\right)}{r^{3}} .
$$

Because $\mathrm{w}_{c}^{\prime}=\infty$, differentiating equation (14b) yields $\mathrm{w}^{\prime \prime \prime}(\hat{r})<0$ for any $\hat{r}$ near $r_{c}$ that satisfies $\mathrm{w}^{\prime \prime}(\hat{r})=0$. Therefore, for all $r$ near $r_{c}$,

$$
\mathrm{w}^{\prime \prime}(r) \geq 0 .
$$

Adding $r A \mathrm{w}^{\prime 3}$ and $-r A \mathrm{w}^{\prime 3}$ to equation (14b) and substituting equations (29) and (31) yield the existence of positive $\eta$ that satisfy

$$
\underline{\lim }_{r \nearrow r_{c}} A \mathrm{w}^{\prime 3}(r)>\eta \text {. }
$$

Consequently, for arbitrarily small positive $\epsilon$ and $r$ sufficiently close to $r_{c}$,

$$
\mathrm{w}^{\prime 3}=\frac{A \mathrm{w}^{\prime 3}}{A}>\frac{\eta}{\epsilon\left(r_{c}-r\right)} .
$$

It follows from equations (30) and (33) that near $r_{c}$,

$$
A^{\prime \prime}>\frac{1}{\left(r_{c}-r\right)^{1 / 3}} .
$$

Integrating equation (34) twice gives, for $r$ near $r_{c}$,

$$
\sqrt{A}>\frac{3}{\sqrt{10}}\left(r_{c}-r\right)^{5 / 6} .
$$

Upon integrating equation (21a) near $r_{c}$ it now follows that $\tau_{c}<\infty$.

Therefore, whenever $\tau_{c}=\infty$, either $\mathrm{w}_{c}=0$ or $\mathrm{w}_{c}^{2}=1$. If $\mathrm{w}_{c}^{2}=1$ and $\Lambda=0$, then, because $\Phi_{c}=0, \lim _{r} \nearrow r_{c} A(r)=1$. Thus, the equations cannot be singular at any finite $r_{c}$. This situation describes particle-like solutions. If $\mathrm{w}_{c}^{2}=1$ and $\Lambda>0$, then, because $\Phi_{c}=0, \Lambda r_{c}^{2}=1$. But in this case, $2 \Lambda r_{c}^{2}>1$ which violates III(c). The only remaining possibility is the desired result; namely that $\mathrm{w}_{c}=0$.

(III $\Rightarrow$ II). If $\Phi_{c}=0$ and $\kappa$ is bounded, then, as above, equation (20) gives $U_{c}=0$. If, in addition, $\mathrm{w}_{c}=0$, then all derivatives of equations (21a) vanish at $\tau_{c}$. Because equations (21a)-(21f) are analytic at any finite $\tau_{c}$, it follows that $\tau_{c}=\infty$. Now II(a) fails to hold only for particle-like solutions. But all such solutions satisfy $\lim _{r}>\infty \mathrm{w}^{2}(r)=1$ which is contrary to the hypotheses. 
(II and III $\Rightarrow$ I). Equation (21f) yields $\lim _{\tau / \infty} \dot{\kappa}(\tau)=0$.

We define

$$
\Theta(\tau)=\operatorname{Arctan} \frac{\dot{\mathrm{w}}}{\mathrm{w}} .
$$

For any solution of equations (21a) $-(21 \mathrm{f}), \Theta$ satisfies the following equation:

$$
\dot{\Theta}=-1+\mathrm{w}^{2} \cos ^{2} \Theta+(2 N-\kappa) \sin \Theta \cos \Theta,
$$

where

$$
\cos \Theta=\frac{\mathrm{w}}{\sqrt{\mathrm{w}^{2}+r^{2} U^{2}}} \quad \text { and } \quad \sin \Theta=\frac{r U}{\sqrt{\mathrm{w}^{2}+r^{2} U^{2}}} .
$$

Standard uniqueness theorems preclude the possibility of a solution smooth at $r=0$ that satisfies, for some $r>0, \mathrm{w}(r)=\mathrm{w}^{\prime}(r)=0$ and $A(r)>0$. Indeed, any such solution must be an extreme Reissner Nordström solution

$$
A=1-\frac{c}{r}+\frac{1}{r^{2}}, \quad \mathrm{w} \equiv 0
$$

which is not smooth at $r=0$.

$\mathrm{II}(\mathrm{b}), \mathrm{III}(\mathrm{c})$, and equation (37) give

$$
\varlimsup_{\tau \nearrow \infty} \dot{\kappa}(\tau)=-1+\sqrt{1-2 \Lambda r_{c}^{2}}<0
$$

in the case $\Lambda>0$. For a proof in the case $\Lambda=0$, see [3]. I follows.

$(\mathrm{I} \Rightarrow \mathrm{II})$. Since $\lim _{r} / r_{c} \mathrm{w}(r)=\mathrm{w}_{c}$ exists for all solutions, equation (14b) easily yields $\mathrm{w}_{c}=0$ for oscillating solutions. Since $\mathrm{w}_{c}^{2}=1$ for particle-like solutions, an oscillating solution cannot be particle-like. Since $r_{c}=\infty$ only for particle-like solutions, $r_{c}$ must be finite for oscillating solutions. This establishes II(a).

To establish II(b) we note that $\overline{\lim }_{\tau / \tau_{c}} \kappa(\tau)<\infty$ [] (or easily from equation (24)) and recall that $\lim _{\tau / \tau_{c}} \kappa(\tau)=-\infty$ whenever $\underline{\lim }_{\tau / \tau \tau_{c}} \kappa(\tau)=-\infty$. When this is the case, there exist $\hat{\tau} \in\left(-\infty, \tau_{c}\right)$ such that $\mathrm{w}(\hat{\tau})>0, \dot{\mathrm{w}}(\hat{\tau})=0$, and for all $\tau \in\left[\hat{\tau}, \tau_{c}\right), \kappa(\tau)<-4$. It follows from equation (37) that $\Theta(\tau) \in(0, \pi / 4)$ throughout the interval $\left(\hat{\tau}, \tau_{c}\right)$; i.e., $\mathrm{w}$ has no zeros in this interval. Since $\mathrm{w}$ is oscillating and analytic for all $\tau \in\left(-\infty, \tau_{c}\right)$, this is impossible.

Therefore, $\kappa$ is bounded throughout the interval $\left(-\infty, \tau_{c}\right)$. If $\left\{\tau_{n}\right\}_{n=0}^{\infty}$ is an increasing sequence that satisfies $\Theta\left(\tau_{n}\right)=n \pi-\pi / 2$, then there exists a sequence $\tilde{\tau}_{n}$ such that $\Theta\left(\tilde{\tau}_{n}\right)=n \pi$ and $\tau_{n}<\tilde{\tau}_{n}<\tau_{n+1}$. Since every term on the right side of equation (37) is bounded, $(r, N, \mathrm{w}, U)$ can be oscillating only if $\lim _{n} / \infty \tau_{n}=\infty$; i.e., only if $\tau_{c}=\infty$.

Alternatively, since $\mathrm{w}$ and $\kappa$ are bounded, $\lim _{\tau / \tau_{c}} U(\tau)$ is well defined if $\tau_{c}$ is finite. Because the solution is oscillating, there exists a sequence $\left\{\tau_{n}\right\} \nearrow \tau_{c}$ that satisfies $U\left(\tau_{n}\right)=\dot{\mathrm{w}} / r=0$; i.e., $U_{c}=0$. Differentiating equation (21a) indefinitely then gives (since $r(\tau)$ is analytic) $r(\tau) \equiv$ constant which is clearly not the case. It follows that $\tau_{c}=\infty$.

Corollary 1. If $\Lambda>1 / 4$, then for no $\lambda$ is the solution $(A(\Lambda, \lambda), \mathrm{w}(\Lambda, \lambda))$ oscillating.

Proof. When $\Lambda>1 / 4$, III cannot hold. Specifically, neither III(c) nor both III(a) and III(b) can hold. 
Corollary 2. If $\Lambda \in(0,1 / 4)$ and $\left(A\left(\Lambda, \lambda_{\text {osc }}\right)\right.$, w $\left.\left(\Lambda, \lambda_{\text {osc }}\right)\right)$ is an oscillating solution, then

$$
r_{c}^{2}\left(\Lambda, \lambda_{\text {osc }}\right)=\frac{1-\sqrt{1-4 \Lambda}}{2 \Lambda} .
$$

Proof. $\Phi_{c}=\mathrm{w}_{c}=0$ implies $r_{c}$ satisfies $1-1 / r^{2}-\Lambda r^{2}=0$. Therefore, $2 \Lambda r_{c}^{2}=$ $1 \pm \sqrt{1-4 \Lambda}$. However, Theorem 1 implies $2 \Lambda r_{c}^{2} \leq 1$. The result follows.

It remains to prove the existence of oscillating solutions. We do so by finding discontinuities in either $r_{c}(\Lambda, \lambda)$ or $\mathrm{w}_{c}(\Lambda, \lambda)$ which we prove can only occur at oscillating solutions. To carry out the proof we first need to establish uniform bounds on $\mathrm{w}_{c}$ for large values of $\lambda$.

As in [8], we define

$$
h(r)=A(r)-\mathrm{w}^{2}(r)
$$

and

$$
g(r)=2 r^{2}-1+\mathrm{w}^{2}(r) .
$$

The next two lemmas and next theorem are adapted from [8].

Lemma 1. Suppose $(A, \mathrm{w})$ is a solution of equations (14a) (14b) with $\Lambda \geq 0$ that satisfies the conditions of equation (16) and the condition $-\mathrm{w}^{\prime \prime}(0)-\lambda>2$. Then $h(r)<0$ as long as $g(r)<0$.

Proof. Simple calculations give $h(0)=0=h^{\prime}(0)=0$, and $h^{\prime \prime}(0)=2 \lambda(1-\lambda)-2 \Lambda / 3$; hence

$$
h(r)<0 \text { for } r \text { near } 0 \text {, if } \lambda>1 .
$$

Moreover, $g(0)=g^{\prime}(0)=0$ and $g^{\prime \prime}(0)=(2-\lambda)$; thus

$$
g(r)<0 \text { for } r \text { near } 0 \text {, if } \lambda>2 .
$$

If $\lambda>1$ and if $\tilde{r}$ is the smallest $r>0$ that satisfies $h(\tilde{r})=0$, then

$$
h^{\prime}(\tilde{r})=\frac{\Phi}{r}-\frac{-2\left(\mathrm{ww}^{\prime}\right)^{2}}{r}-2 \mathrm{ww}^{\prime} .
$$

Considering the right side of equation (41) as a quadratic form in $s=\left(\mathrm{ww}^{\prime}\right)$ gives discriminant at $\tilde{r}$

$$
\begin{aligned}
\Delta & =4\left(1+\frac{2 \Phi}{r^{2}}\right)=4\left[1+\frac{2(1-A)}{r^{2}}-\frac{2\left(1-\mathrm{w}^{2}\right)^{2}}{r^{4}}-2 \Lambda\right] \\
& =4\left[1+\frac{2\left(1-\mathrm{w}^{2}\right)}{r^{2}}-\frac{2\left(1-\mathrm{w}^{2}\right)^{2}}{r^{4}}-2 \Lambda\right] \\
& =4\left[1+\frac{2\left(1-\mathrm{w}^{2}\right)}{r^{2}}\left(1-\frac{1-\mathrm{w}^{2}}{r^{2}}\right)-2 \Lambda\right] \\
& <4\left[1-\frac{2\left(1-\mathrm{w}^{2}\right)}{r^{2}}-2 \Lambda\right]<4(1-4-2 \Lambda)<0
\end{aligned}
$$

whenever $g(\tilde{r})<0$. Since $-(2 / \tilde{r}) s^{2}-2 s+\Phi / \tilde{r}<0$ for large negative $s$, it follows that $h(r)<0$ as long as $g(r)<0$.

Lemma 2. Suppose $(A, \mathrm{w})$ is a solution of equations (14a) (14b) with $\Lambda \geq 0$ that satisfies the conditions of equation (16) and the condition $-\mathrm{w}^{\prime \prime}(0)=\lambda>2$. Then $g^{\prime}(r)<0$ as long as $h(r)<0$. 
Proof. For any solution $(A, \mathrm{w})$ of equations (14a) $-14 \mathrm{~b}), g$ satisfies the following equation:

$$
g^{\prime \prime}(r)=-\frac{\Phi}{r A} g^{\prime}+\frac{2}{r^{2} A}\left[r^{2} A \mathrm{w}^{\prime 2}-\left(1-\mathrm{w}^{2}\right)^{2}-2 \Lambda r^{4}+g\right] .
$$

If $\lambda>2$ and $\tilde{r}$ is the smallest $r$ that satisfies $g^{\prime}(r)=0$, then

$$
\begin{aligned}
g^{\prime \prime}(\tilde{r}) & =\frac{2}{\tilde{r}^{2} A}\left[r^{2} A \mathrm{w}^{\prime 2}-\left(1-\mathrm{w}^{2}\right)^{2}-2 \Lambda r^{4}+g\right]_{r=\tilde{r}} \\
& \leq \frac{2}{\tilde{r}^{2} A}\left[r^{2} \mathrm{w}^{2} \mathrm{w}^{\prime 2}-\left(1-\mathrm{w}^{2}\right)^{2}-2 \Lambda r^{4}+g\right]_{r=\tilde{r}} \\
& =\frac{2}{\tilde{r}^{2} A}\left[4 \tilde{r}^{4}-\left(1-\mathrm{w}^{2}\right)^{2}-2 \Lambda r^{4}+g\right]_{r=\tilde{r}} \\
& =\frac{2}{\tilde{r}^{2} A}\left[g\left(1+2 \tilde{r}^{2}+\left(1-\mathrm{w}^{2}\right)\right)-2 \Lambda r^{4}\right]_{r=\tilde{r}}<0
\end{aligned}
$$

whenever $h(\tilde{r})$ and $g(\tilde{r})$ are negative. The result follows.

Theorem 2. Suppose $(A, \mathrm{w})$ is a solution of equations (14a) (14b) with $\Lambda \geq 0$ that satisfies the conditions of equation (16) and the condition $-\mathrm{w}^{\prime \prime}(0)=\lambda>2$. Then $0<\mathrm{w}(r)<1$ for all $r \in\left[0, r_{c}\right)$.

Proof. $h$ and $g$ are both negative near 0 . Therefore, if there exists some $\hat{r} \in\left(0, r_{c}\right)$ that satisfies $\mathrm{w}(\hat{r})=0$, then $h(\hat{r})>0$. In this case, there exists a smallest $r$, say $r_{1}$, that satisfies $h\left(r_{1}\right)=0$. Similarly, $\mathrm{w}(\hat{r})$ gives $g^{\prime}(\hat{r})>0$. Consequently, there exists a smallest $r$, say $r_{2}$, that satisfies $g^{\prime}\left(r_{2}\right)=0$. Now, Lemma 1 gives $r_{1}<r_{2}$. Also, Lemma 2 gives $r_{1}>r_{2}$. Both cannot hold. The result follows.

The following corollary, besides being used to prove Theorem 3, is interesting in its own right.

Corollary 3. Suppose $(A, \mathrm{w})$ is a solution of equations (14a 14b with $\Lambda \geq 0$ that satisfies the conditions of equation (16) and the condition $-\mathrm{w}^{\prime \prime}(0)=\lambda>2$. Then $r_{c}<1 / \sqrt{2}$.

Proof. If $r_{c} \geq 1 / \sqrt{2}$, then $g\left(r_{c}\right)>0$. It follows that there exists a smallest $r_{2}$ that satisfies $g^{\prime}\left(r_{2}\right)=0$. Lemma 1 gives $h(r)<0$ for all $r<r_{2}+\delta_{1}$ for some small positive $\delta_{1}$. Lemma 2 then gives $g^{\prime}(r)<0$ for all $r<r_{2}+\delta_{2}$ for some small positive $\delta_{2}$, contradicting the fact that $g^{\prime}\left(r_{2}\right)=0$. The result follows.

Lemma 3. Suppose $(\widetilde{\Lambda}, \tilde{\lambda})$ is such that $\tau_{c}(\widetilde{\Lambda}, \tilde{\lambda})<\infty$ and $\kappa_{c}^{2}(\tilde{\Lambda}, \tilde{\lambda})<\infty$ both hold. Then $\tau_{c}(\Lambda, \lambda), r_{c}(\Lambda, \lambda)$, and $\mathrm{w}_{c}(\Lambda, \lambda)$ are continuous at $(\tilde{\Lambda}, \tilde{\lambda})$.

Proof. For all solutions of equations (21a)-(21f), $\mathrm{w}_{c}^{2}<\infty$ [7]. Therefore, for the solution corresponding to $(\widetilde{\Lambda}, \tilde{\lambda})$, equations (21a) $-(21 \mathrm{f})$ are nonsingular at $\tau_{c}$. Therefore, the solution $(r, N, \mathrm{w}, U)(\tau)$ is analytic in $\tau \in\left(-\infty, \tau_{c}+\delta\right)$ for some $\delta>0$. In particular, all variables have finite limits at $\tau_{c}$.

We consider first the case $U_{c}=0$. If $\mathrm{w}_{c}\left(1-\mathrm{w}_{c}^{2}\right)=0$, then $d^{n} r\left(\tau_{c}\right) / \mathrm{d} \tau^{n}=0$ for all $n>0$. Since $r(\tau)$ is analytic, this implies $r(\tau)=r_{c}$ for all $\tau \in\left(-\infty, \tau_{c}\right)$, which is clearly false. Therefore, $\mathrm{w}_{c}\left(1-\mathrm{w}_{c}^{2}\right) \neq 0$. Now, differentiating equation (21c) twice yields $\dot{N}=\ddot{N}=0$ whereas $\frac{\mathrm{d}^{3}}{\mathrm{~d} \tau^{3}} N\left(\tau_{c}\right)=-\left[\mathrm{w}\left(1-\mathrm{w}^{2}\right)\right]^{2} / r^{2}<0$. Therefore, $N(\tau)<0$ in a neighborhood $\left(\tau_{c}(\widetilde{\Lambda}, \tilde{\lambda}), \tau_{c}(\widetilde{\Lambda}, \tilde{\lambda})+\epsilon\right)$, where $\epsilon<\delta$ is arbitrarily small. By continuous dependence of solutions on parameters, there exists a neighborhood 
$V \subset[0, \infty) \times \mathbb{R}$ of $(\tilde{\Lambda}, \tilde{\lambda})$ such that for all $(\Lambda, \lambda) \in V, N(\Lambda, \lambda, \tau)>0$ for all $\tau \in\left(-\infty, \tau_{c}(\tilde{\Lambda}, \tilde{\lambda})-\epsilon\right)$ and $N(\Lambda, \lambda, \tau)<0$ for all $\tau>\tau_{c}(\tilde{\Lambda}, \tilde{\lambda})+\epsilon . \quad N(\Lambda, \lambda, \tau)$ continuous gives $\tau_{c}(\Lambda, \lambda) \in\left(\tau_{c}(\tilde{\Lambda}, \tilde{\lambda})-\epsilon, \tau_{c}(\tilde{\Lambda}, \tilde{\lambda})+\epsilon\right)$. Since $\epsilon$ is arbitrary, it follows that $\tau_{c}(\Lambda, \lambda)$ is continuous.

A similar argument gives the continuity of $\tau_{c}$ in the case $U_{c} \neq 0$. Alternatively, continuity of $\tau_{c}$ in the case $U_{c} \neq 0$ follows from the Implicit Function Theorem applied to $N(\Lambda, \lambda, \tau)$.

If $x(\Lambda, \lambda, \tau)=y(\tau, r(\Lambda, \lambda, \tau), N(\Lambda, \lambda, \tau), \mathrm{w}(\Lambda, \lambda, \tau), U(\Lambda, \lambda, \tau))$, where $y: \mathbb{R}^{5} \rightarrow \mathbb{R}$ is smooth but otherwise arbitrary, then

$$
x_{c}(\Lambda, \lambda)=y\left(\tau_{c}(\Lambda, \lambda), r\left(\Lambda, \lambda, \tau_{c}(\Lambda, \lambda)\right), 0, \mathrm{w}\left(\Lambda, \lambda, \tau_{c}(\Lambda, \lambda)\right), U\left(\Lambda, \lambda, \tau_{c}(\Lambda, \lambda)\right)\right) .
$$

Continuous dependence on parameters and the continuity of $\tau_{c}$ give $x_{c}(\Lambda, \lambda)$ is continuous. In particular, $r_{c}$ and $\mathrm{w}_{c}$ are continuous.

Lemma 4. For any $\alpha>0$ and $\beta>1$, the sets $\left\{(\Lambda, \lambda) \in[0, \infty) \times \mathbb{R} \mid r_{c}(\lambda, \lambda)>\alpha\right\}$ and $\left\{(\Lambda, \lambda) \in[0, \infty) \times \mathbb{R} \mid \mathrm{w}_{c}^{2}(\Lambda, \lambda)>\beta\right\}$ are open.

Proof. Continuous dependence on parameters implies $\left\{(\Lambda, \lambda) \in[0, \infty) \times \mathbb{R} \mid r_{c}(\Lambda, \lambda)\right.$ $>\alpha\}$ is open. It follows easily from equation (14b) that whenever $\mathrm{w}^{2}(r)>1$ and $\mathrm{w}^{\prime}(r)=0$, then $\mathrm{ww}^{\prime \prime}(r)>0$. This and continuous dependence give $\{(\Lambda, \lambda) \in$ $\left.[0, \infty) \times \mathbb{R} \mid \mathrm{w}_{c}^{2}(\Lambda, \lambda)>\beta\right\}$ is open.

Theorem 3. For each $\Lambda \in[0,1 / 4)$, there exists at least one $\lambda_{\text {osc }}(\Lambda) \in[0,2]$ such that the solution $\left(A\left(\Lambda, \lambda_{\text {osc }}\right), \mathrm{w}\left(\Lambda, \lambda_{\text {osc }}\right)\right)$ of equations (14a)-(14b) is an oscillating solution.

Proof. We define the set $\mathcal{G} \subset \mathbb{R}^{2}$ as follows: $(\Lambda, \lambda) \in \mathcal{G}$ whenever the corresponding solution of equations (14a)-(14b) (or the equivalent equations (21a)-(21f) satisfies the following:

(1) $r_{c}<\infty$

(2) $\kappa(\tau)>-1$ for all $\tau \in\left(-\infty, \tau_{c}\right)$,

(3) $\mathrm{w}^{2}(r)<2$ for all $r \in\left(0, r_{c}\right)$.

If, for any $(\Lambda, \lambda) \in \mathcal{G}, \tau_{c}<\infty$, then the conditions of Lemma 3 are satisfied. Lemma 3 and continuous dependence on parameters then yield an open neighborhood $V \subset \mathcal{G}$ of $(\Lambda, \lambda)$ such that $r_{c}$ and $\mathrm{w}_{c}$ are continuous in $V$. Considering the contrapositive, we have $\tau_{c}\left(\Lambda_{\text {osc }}, \lambda_{\text {osc }}\right)=\infty$ at any $\left(\lambda_{\text {osc }}, \lambda_{\text {osc }}\right)$ in $\mathcal{G}$ where either $r_{c}$ or $\mathrm{w}_{c}$ is discontinuous. In light of Theorem 1, to complete the proof we need only to find such $(\Lambda, \lambda)$.

First, we note that $\left\{(\Lambda, \lambda) \in \mathcal{G} \mid \tau_{c}(\Lambda, \lambda)<\infty\right\}$ is nonempty. Indeed, it follows from equation (21f) and Corollary 3 that if $\Lambda \in[0,1)$ and $\lambda>2$, then $(\Lambda, \lambda) \in \mathcal{G}$. Furthermore, Theorem 2 states that for any such $(\Lambda, \lambda)$, the corresponding solution $(A(\lambda, r), \mathrm{w}(\lambda, r))$ satisfies $0<\mathrm{w}(r)<1$ for all $r \in\left(0, r_{c}\right]$. Corollary 3 gives $r_{c}<1$. Theorem 1 gives $\tau_{c}<\infty$. Indeed, $\tau_{c}=\infty$ implies $\mathrm{w}_{c}=0$ or $\mathrm{w}_{c}=1$; neither of which is the case.

This establishes that for any $(\Lambda, \lambda) \in[0,1) \times(2, \infty)$, the following hold:

(1) $r_{c}(\Lambda, \lambda)<1$,

(2) $\mathrm{w}_{c}(\Lambda, \lambda) \in(0,1)$

(3) $\kappa_{c}(\Lambda, \lambda) \geq 0$

(4) $\tau_{c}(\Lambda, \lambda)<\infty$. 
For any $\bar{\Lambda}<1 / 4$, we define

$$
\begin{aligned}
& \lambda_{\text {discont }}^{r}=\inf \left\{\lambda \in(0, \infty) \mid r_{c}(\bar{\Lambda}, \mu) \text { is continuous for all } \mu \in(\lambda, \infty)\right\}, \\
& \lambda_{\text {discont }}^{\mathrm{w}}=\inf \left\{\lambda \in(0, \infty) \mid \mathrm{w}_{c}(\bar{\Lambda}, \mu) \text { is continuous for all } \mu \in(\lambda, \infty)\right\},
\end{aligned}
$$

and

$$
\lambda_{\text {osc }}=\max \left\{\lambda_{\text {discont }}^{r}, \lambda_{\text {discont }}^{\mathrm{w}}\right\} .
$$

Both $\lambda_{\text {discont }}^{r}$ and $\lambda_{\text {discont }}^{\mathrm{w}}$ are well defined because of Lemma 3 and the four statements satisfied by $(\Lambda, \lambda) \in[0,1) \times(2, \infty)$. We now assume that $\lambda_{o s c}=0$ and arrive at a contradiction. We define

$$
\begin{aligned}
\lambda_{r} & =\sup \left\{\lambda \in(0, \infty) \mid r_{c}(\bar{\Lambda}, \lambda)=\sqrt{2}\right\}, \\
\lambda_{\mathrm{w}} & =\sup \left\{\lambda \in(0, \infty) \mid \mathrm{w}_{c}^{2}(\bar{\Lambda}, \lambda)=1\right\} .
\end{aligned}
$$

The unique solution $(A(\bar{\Lambda}, 0), \mathrm{w}(\bar{\Lambda}, 0))$ is that of deSitter space

$$
A=1-\frac{\Lambda r^{2}}{3}, \quad \mathrm{w} \equiv 1
$$

in the case $\Lambda>0$ or Minkowski space

$$
A \equiv 1, \quad \mathrm{w} \equiv 1
$$

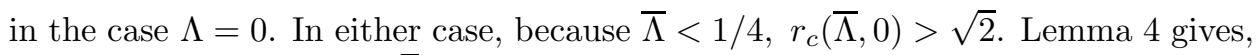
for small $\lambda, r_{c}(\bar{\Lambda}, \lambda)>\sqrt{2}$. Therefore, because by assumption $\lambda_{\text {discont }}^{r}=0, \lambda_{r}$ is well defined.

$$
\Phi_{c}\left(\bar{\Lambda}, \lambda_{r}\right)=1-\frac{\left(1-\mathrm{w}_{c}^{2}\right)^{2}}{r_{c}^{2}}-\bar{\Lambda} r_{c}^{2}>1-\frac{\left(1-\mathrm{w}_{c}^{2}\right)^{2}}{2}-\frac{1}{2} .
$$

$\Phi_{c} \leq 0$ for any solution forces $\mathrm{w}_{c}^{2}\left(\bar{\Lambda}, \lambda_{r}\right)>2$. Since by assumption $\mathrm{w}_{c}(\bar{\Lambda}, \lambda)$ is continuous for all $\lambda$ and $\mathrm{w}_{c}^{2}(\bar{\Lambda}, 3)<1$, it follows that $\lambda_{\mathrm{w}}$ is well defined. Now on one hand equation (45) gives $\lambda_{\mathrm{w}}>\lambda_{r}$. But on the other hand, since $\lambda_{\mathrm{w}}>$ $\lambda_{r}, r_{c}\left(\bar{\Lambda}, \lambda_{\mathrm{w}}\right)<\sqrt{2}$ from which it follows that

$$
\Phi_{c}\left(\bar{\Lambda}, \lambda_{\mathrm{w}}\right)=1-\frac{\left(1-\mathrm{w}_{c}^{2}\right)^{2}}{r_{c}^{2}}-\bar{\Lambda} r_{c}^{2}>1-\frac{1}{2}>0 .
$$

However, $\Phi_{c} \leq 0$. This is a contradiction. We conclude that $\lambda_{o s c}>0$. By construction, one of $r_{c}$ or $\mathrm{w}_{c}$ is discontinuous at $\left(\bar{\Lambda}, \lambda_{\text {osc }}\right)$. It remains to prove that $\left(\bar{\Lambda}, \lambda_{\text {osc }}\right) \in \mathcal{G}$.

The definition of $\lambda_{o s c}$ and Lemma四 give $\mathrm{w}_{c}^{2}\left(\bar{\Lambda}, \lambda_{o s c}\right) \leq 1$ and $r_{c}\left(\bar{\Lambda}, \lambda_{o s c}\right) \leq \sqrt{2}$. $\bar{\Lambda}<1 / 4$ then gives $2 \Lambda r_{c}^{2}\left(\bar{\Lambda}, \lambda_{\text {osc }}\right)<1$. Equation (21f) easily yields $\kappa_{c}\left(\bar{\Lambda}, \lambda_{\text {osc }}\right) \geq 0$. This completes the proof.

Corollary 4. There exists at least one $\lambda_{\text {osc }} \in[0,2]$ such that the solution of equations (14a) -14b) with $\Lambda=1 / 4$ that satisfies the conditions of equation (16) with $\mathrm{w}^{\prime \prime}(0)=-\lambda_{\text {osc }}$ is an oscillating solution. Moreover, for this solution, $r_{c}=\sqrt{2}$.

Proof. We define $\mathcal{O} \subset \mathbb{R}^{2}$ by

$$
\mathcal{O}=\{(\Lambda, \lambda) \in[0,1 / 4) \times[0, \infty) \mid(A(\Lambda, \lambda), \mathrm{w}(\Lambda, \lambda)) \text { is oscillating }\} .
$$

Because of Theorem 1 and Theorem $2, \mathcal{O} \subset[0,1 / 4) \times[0,2]$. We define $\mathcal{F} \subset \mathcal{G}$ by

$$
\mathcal{F}=\left\{(\Lambda, \lambda) \in \mathcal{G} \mid \tau_{c}(\Lambda, \lambda)<\infty\right\} .
$$

Now, Lemma 3 gives $\mathcal{F}$ is open in $\mathbb{R}^{2}$. Theorem 1 gives $\mathcal{O} \subset \mathcal{F}^{\prime}$ (the complement of $\mathcal{F}$ in $\mathbb{R}^{2}$ ). Therefore, $\overline{\mathcal{O}}$ (the closure of $\mathcal{O}$ in $\mathbb{R}^{2}$ ) satisfies $\overline{\mathcal{O}} \cap \mathcal{F}$ is empty. 
Invoking Theorem 3] we consider a sequence $\left\{\left(\Lambda_{n}, \lambda_{n}\right)\right\}_{n=0}^{\infty}$ such that $\Lambda_{n} \nearrow 1 / 4$ and, for each $n,\left(\Lambda_{n}, \lambda_{n}\right)$ is oscillating. Since $\left\{\left(\Lambda_{n}, \lambda_{n}\right)\right\}_{n=0}^{\infty} \subset[0,1 / 4] \times[0,2]$ and $[0,1 / 4] \times[0,2]$ is compact, it follows that $(\{1 / 4\} \times[0,2]) \cap \overline{\mathcal{O}}$ is nonempty. We denote any point in this intersection by $\left(1 / 4, \lambda_{\text {osc }}\right)$. Corollary 2 and Lemma 4 imply $r_{c}\left(1 / 4, \lambda_{\text {osc }}\right) \leq \sqrt{2}$. Equation (21f) yields $\kappa_{c}\left(1 / 4, \lambda_{\text {osc }}\right) \geq 0$. Lemma 3 then gives $\tau_{c}\left(1 / 4, \lambda_{\text {osc }}\right)=\infty$. Theorem 1 gives $\left(A\left(1 / 4, \lambda_{\text {osc }}\right), \mathrm{w}\left(1 / 4, \lambda_{\text {osc }}\right)\right)$ is oscillating. Since $\Phi_{c}\left(1 / 4, \lambda_{\text {osc }}\right)=\mathrm{w}_{c}\left(1 / 4, \lambda_{\text {osc }}\right)=0$, it follows that $r_{c}\left(1 / 4, \lambda_{\text {osc }}\right)=\sqrt{2}$.

\section{Concluding Remarks}

We conclude with the following remarks:

(1) Since an oscillating spacetime is static and spherically symmetric, it is geodesically complete if and only if, for arbitrary $t_{0}, \phi_{0}$, and $\theta_{0}$, the curve $\gamma(\tau)=$ $\left(t_{0}, r(\tau), \phi_{0}, \theta_{0}\right), \tau \in(-\infty, \infty)$, has infinite length. It follows easily from metric (22) that this is the case. Therefore, the spacetime covered by the single chart described by $\mathbb{R} \times\left[0, r_{c}\right) \times S^{2}$ with $\{0\} \times S^{2}$ identified should be viewed as consisting of all of spacetime.

(2) As emphasized in the introduction, the difference between oscillating spacetimes and particle-like spacetimes is geometric. Indeed, oscillating spacetimes are not asymptotically flat. This can be seen in many ways. For example, we take the orthonormal frame

$$
e_{0}=\frac{1}{c C \sqrt{A}} \frac{\partial}{\partial t}, \quad e_{1}=\sqrt{A} \frac{\partial}{\partial r}, \quad e_{2}=\frac{1}{r} \frac{\partial}{\partial \phi}, \quad e_{3}=\frac{1}{r \sin \phi} \frac{\partial}{\partial \theta} .
$$

The sectional curvature $K_{23}$ in the plane determined by $e_{2}$ and $e_{3}$ satisfies

$$
K_{23}=\frac{(1-A)}{r^{2}}
$$

which approaches $1 / r_{c}^{2}$ as $r \nearrow r_{c}$. $\lim _{r} \nearrow r_{c} K_{23} \neq 0$ as it must for an asymptotically flat space.

(3) Generic, noncompact, and oscillating solutions all have different types of singularities at $r_{c}$. They also determine spacetime manifolds that have different global geometries. In a separate analysis we show to what extent the global geometry of the underlying spacetime manifold is determined by the nature of the singularity in the solution at $r=r_{c}$.

\section{REFERENCES}

[1] R. Bartnik and J. McKinnon, Particlelike solutions of the Einstein-Yang-Mills equations, Physical Review Letters, 61 (2), 1988. MR89e:83015

[2] R. Bartnik, The spherically symmetric Einstein Yang-Mills equations, Relativity TodayProceedings of the third Hungarian Relativity Workshop, pages 221-240, 1989.

[3] P. Breitenlohner, P. Forgács, and D. Maison, Static spherically symmetric solutions of the Einstein-Yang-Mills equations, Communications in Mathematical Physics, 163 141-172, 1994. MR95b:83021

[4] A. Linden, Existence of noncompact static spherically symmetric solutions of Einstein $\mathrm{SU}(2)$ Yang-Mills equations, Communications in Mathematical Physics, 221 (3) 525-547, 2001. MR2002f:53040

[5] A. Linden, Far field behavior of noncompact static spherically symmetric solutions of Einstein SU(2)-Yang-Mills equations, Journal of Mathematical Physics, 42 (3) 1196-1201, 2001. MR2002d:53104

[6] A. Linden, Near field behavior of static spherically symmetric solutions of Einstein $\mathrm{SU}(2)$ Yang-Mills equations with cosmological constant, Michigan Mathematics Journal, 50 (1) 201224, 2002. MR2003g:83021 
[7] A. Linden, Horizons in spherically symmetric static Einstein SU(2)-Yang-Mills spacetimes, Classical and Quantum Gravity, 18 (4) 695-708, 2001. MR2002f:53040

[8] J. Smoller, A. Wasserman, S.-T. Yau, and J. B. McLeod, Smooth static solutions of the Einstein/Yang-Mills equations, Communications in Mathematical Physics, 143 115-147, 1991. MR93a:58044

[9] J. Smoller and A. Wasserman, Existence of infinitely many smooth static solutions of the Einstein/Yang-Mills equations, Communications in Mathematical Physics, 151 (2) 303-325, 1993. MR94a:58042

[10] J. Smoller and A. Wasserman, Regular solutions of the Einstein-Yang-Mills equations, Journal of Mathematical Physics, 36 (8), 4301-4323, August 1995. MR96g:53037

[11] M. S. Volkov, N. Straumann, G. Lavrelashvili, M. Heusler, and O. Brodbeck, Cosmological analogues of the Bartnik-McKinnon solutions, Physical Reviews D, 54, 7243-7251, 1996.

School of Mathematics and Statistics, University of Canberra, Australian Capital Territory \#2601, Australia

E-mail address: lindena_9@hotmail.com 